\title{
Sclerosing stromal tumour of the ovary: a case report
}

M. V. C. de Silva ${ }^{1}$ and L. R. Amarasekera ${ }^{2}$

The Ceylon Journal of Medical Science 1996; 39: 57-59

\section{Summary}

A case of sclerosing stromal tumour of the ovary occurring in a nineteen year old female is presented. The clinical presentation, diagnostic pathological features, biological behaviour and differential diagnosis of this rare tumour are discussed.

Key words: Ovary, Sclerosing stromal tumour.

\section{Introduction}

Sclerosing stromal tumour is a distinctive type of a rare stromal cell tumour of the ovary. It differs in clinical and pathological features from other ovarian stromal tumours such as fibromas and thecomas. Fibromas and thecomas have a peak incidence in the 5 th or 6 th decade of life, whereas sclerosing stromal tumours occur in the second and third decades $(1,2)$. In contrast to thecomas the sclerosing stromal tumour has been associated with oestrogen secretion in only a few cases. Reports of androgen secretion either alone or together with oestrogen is even more uncommon $(3,4)$. All sclerosing tumours reported to date have been benign (1).

This is the first documentation of this tumour in Sri Lanka.

\section{Case report}

A nineteen year old female presented with colicky pain in the left, lower abdomen of two days duration. Her last menstrual period had been 36 days prior to the onset of abdominal pain. She complained of irregular menstrual periods. An ultrasound scan showed a solid and cystic mass, $8 \mathrm{~cm}$ in diameter, in the left iliac fossa. At laporotomy a left ovarian cystic mass was found twisted around the left fallopian tube. The tumour appeared to be viable with no evidence of necrosis. The right ovary appeared normal. The uterus was normal in size and shape. A small amount of serous fluid was found in the pelvic cavity. The patient made an uneventful recovery and there has been no recurrence to date.

\section{Pathology}

The macroscopy specimen consisted of an encapsulated tumour measuring $6 \times 4 \times 3 \mathrm{~cm}$ with adherent fallopian tube. There was no identifiable normal ovarian tissue. The cut surface appeared variegated with oedematous, mucoid, cystic and solid white areas.

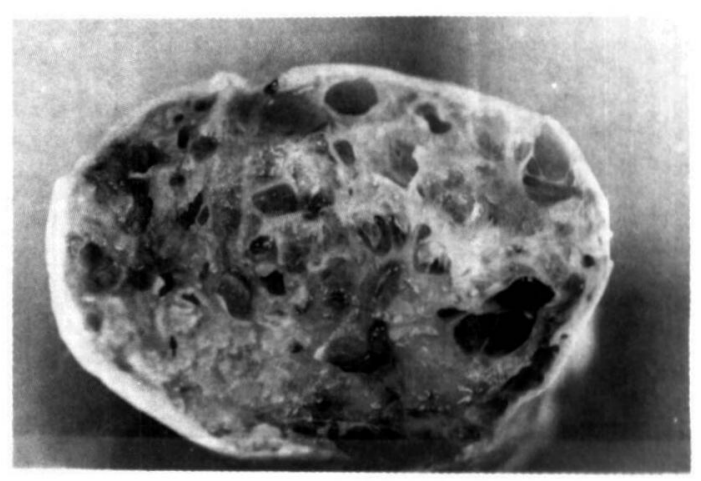

Fig. 1. Solid, cystic and oedematous cut surface of the tumour.

Microscopic examination showed a pseudolobular pattern in which cellular nodules were separated by less cellular oedematous connective tissue. The cellular nodules were composed of fibroblasts, rounded vacuolated cells, and thin walled variably sized irregular vessels. Fat stains confirmed the presence of lipid droplets in the rounded cells. Sclerosis was evident within some nodules. There was no evidence of pleomorphism or mitotic figures. 
The cysts seen macroscopically are due to collection of oedema fluid in between the solid areas of the tumour.

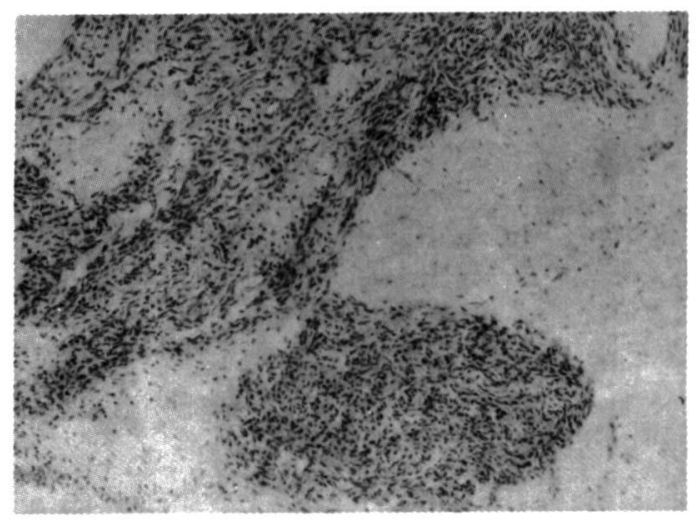

Fig. 2. Pseudolobular appearance with cellular nodules and oedematous less cellular areas $(x 100)$

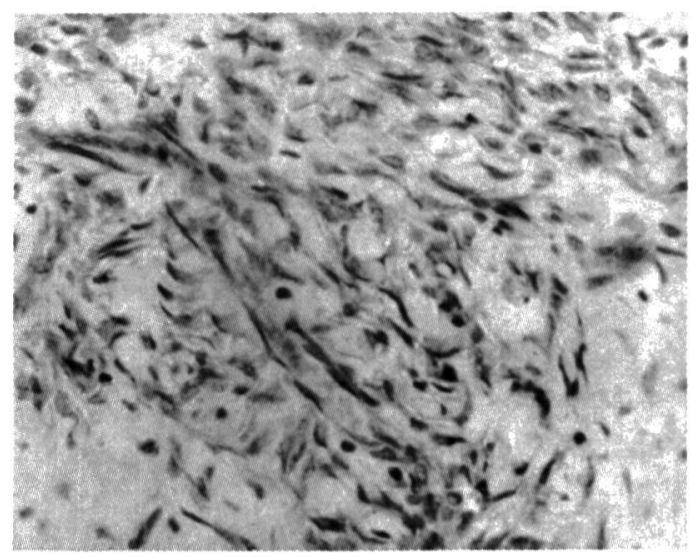

Fig. 3. Spindle shaped fibroblasts within the cellular areas $(x 400)$

\section{Discussion}

Majority of sclerosing stromal tumours present with a history of irregular menstrual bleeding or chronic pelvic pain (5). The acute presentation of this patient due to twisting of the tumour was unusual.

Secretion of oestrogens and/or androgens by sclerosing stromal tumours has been reported in a few cases only. Endocrine studies could not be performed in this patient due to the acute presentation.
The macroscopic variegated appearance of this tumour contrasts with the homogenous yellowish white cut surface of thecomas and the white whorled cut surface of fibromas. The tumour can be diagnosed on the microscopic appearance of sections stained with haemotoxylin and eosin. The most characteristic feature which permits its distinction from a fibroma, is the heterogeneous low power appearance with alternating cellular and oedematous areas $(1,5)$. The presence of ectatic vessels and a dual population of fibroblasts and vacuolated cells within the cellular nodules are useful diagnostic features. Hyaline plaques which are commonly found in thecomas and fibromas are rarely seen in sclerosing stromal tumours. Occasionally the vacuolated cells may have a signet-cell appearance, creating confusion with Krukenberg tumours, but the latter will contain mucin instead of lipid.

It is also important to differentiate this tumour from a benign lesion called " massive oedema of the ovary", which also occurs predominantly in a younger age group. In the latter condition normal ovarian structures such as follicles, corpora lutea and albicancia can be identified within the oedematous stroma (5).

As the sclerosing stromal tumour differs from the more common fibromas and thecomas in the clinical presentation, biological behaviour and pathological features, it should be regarded as a distinct type of a stromal cell tumor.

\section{References}

1. Young RH, Scully RE. Sex cord stromal, steroid cell and other ovarian tumours with endocrine, paraendocrine and paraneoplastic manifestations. In: Kurman RJ ed. Blaustein's Pathology of the Female Genital Tract. 4th edition. New York. Sp inger-Verlag, 1994; 801-802.

2. Young RH, Scully RE. Ovarian sex cord stromal tumours: Recent progress. International Journal of Gynaecological Pathology. 1992; 1:101 
3. Ismail SM, Walker SM. Bilateral virilizing sclerosing stromal tumours of the ovary in a pregnant woman with Gorlin's syndrome: implications for pathogenesis of ovarian

5. Chalvardjian A, Scully RE. Sclerosing stromal tumours of the ovary. Cancer. $1973 ; 31$ : 664-670.

stromal neoplasms. Histopathology. 1990; 17: 159-163.

4. Quinn MA, Oster AO, Fortune D, Hudson B. Sclerosing stromal tumour of the ovary, case report with endocrine studies. British Journal of Obstetrics and Gynaecology. $1981 ; 88$ : 555-558. 